\title{
Table ronde 1
}

\section{État des lieux après \\ 10 ou 15 ans d'actions \\ pour les maladies rares}

Participent à la table ronde :

Christian Cottet, AFM-Téléthon

Hélène Dollfus, CHRU de Strasbourg

Sylvain Forget, Nassyane

Marc Hanauer, Orphanet

d'éléments positifs, liés notamment à la structuration de l'organisation sur le territoire national. Même si l'appel d'offres a été décalé de décembre 2015 à 2016, des centres de référence se mettent en place au niveau européen. Le modèle français est donc en train d'être décliné à l'échelle du continent.

\section{Point de vue des associations de malades}

\section{Christian Cottet \\ Mon intervention s'articulera en quatre temps.}

\section{0 ou 15 ans d'actions concertées}

Certaines associations de malades mènent depuis un demi-siècle des actions militantes dans lesquelles s'enracine depuis 2004 la politique concertée de santé publique pour les maladies rares. Le mouvement associatif des maladies rares, qui s'est développé en France à la fin des années 1950, a fortement contribué aux conditions du progrès thérapeutique et médical. Les associations ont développé une vision, élaboré une stratégie, mis en place des organisations, construit des alliances avec le monde scientifique et le monde médical ; en outre, elles ont fait connaître les maladies et ont interpellé les pouvoirs publics, constituant ainsi une force solidaire organisée et efficace au service de l'intérêt général.

Au début des années 2000, la problématique des maladies rares, identifiée comme une question de santé publique, a commencé à s'imposer en France sous l'impulsion des associations. Il était alors fondamental de mettre en place une stratégie nationale volontariste, définie comme une politique prioritaire et pilotée nationalement en réponse aux enjeux identifiés tant nationaux qu'européens. Cette priorité s'est concrétisée dans le PNMRI, inscrit dans la loi de santé publique de 2004. Il a permis d'améliorer l'accès au diagnostic et la prise en charge des personnes atteintes de maladies rares à travers la mise en place des centres de référence et de compétences.

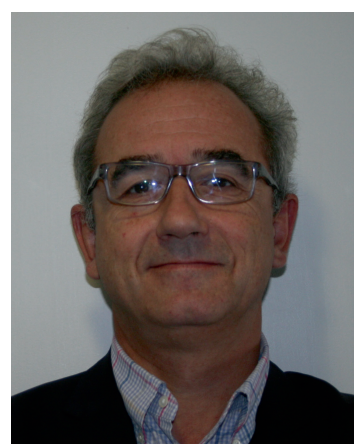

La table ronde est animée par Didier Lacombe (Fédération Française de Génétique Humaine)

Au terme du PNMRl, il a fallu une forte mobilisation des associations pour que l'effort de la puissance publique soit poursuivi dans un deuxième plan lancé en février 2011. Pour le mouvement associatif, le PNMR2 était, à son lancement, en demi-teinte par son manque d'ambitions et de moyens.

\section{Bilan et constats}

Les deux premiers PNMR ont apporté une amélioration réelle. Cependant, beaucoup reste encore à faire parce que les attentes légitimes des malades n'ont pas toutes été prises en compte.

Des progrès ont été réalisés dans le diagnostic ; pour autant, l'errance diagnostique demeure une réalité pour nombre de familles. Ainsi, $10 \%$ de situations d'errance ou d'impasse diagnostique ont été recensées pour les familles avec atteinte neuromusculaire, qui sont pourtant suivies dans les centres de référence.

Les centres de référence ont permis le regroupement et l'articulation des expertises - c'est un réel progrès.

La question de l'adaptation de la tarification à l'activité (T2A) au financement des consultations complexes et pluridisciplinaires reste un point d'achoppement.

Malgré l'objectif ambitieux du PNMRl, moins de 80 Protocoles nationaux de diagnostic et de soins (PNDS) ont à ce jour été publiés.

Des moyens ont été apportés pour des équipements en séquenceurs de moyen et haut débit. Toutefois, la plate-forme nationale de séquençage à très haut débit est toujours en attente. De plus, l'accès à ces méthodes de diagnostic pointues et leur prise en charge doivent encore être organisés. 
La Fondation maladies rares a été créée pour développer et coordonner l'effort de recherche, mais les financements publics alloués à cette structure font défaut.

Les enjeux du développement thérapeutique n'ont pas été suffisamment traités, notamment dans le domaine des médicaments de thérapie innovante. Le lien entre la santé, la recherche publique et le monde industriel n'a pas été suffisamment pris en compte.

La réforme de la politique du médicament s'est faite en préservant la spécificité des maladies rares. Cependant, il reste encore beaucoup à faire sur la question des utilisations hors AMM.

La BNDMR a été développée laborieusement, mais la problématique des registres et des bases de données pour la recherche n'avance pas vite. De plus, de nombreux projets sont bloqués faute de moyens.

Le PNMR définissait assez timidement des orientations pour l'articulation entre les priorités nationales et les territoires de santé. Force est de constater que les plans régionaux de santé contiennent très peu d'éléments sur ce sujet.

Enfin, les FSMR sont une avancée très pertinente en termes de structuration de l'écosystème. Mais leur lancement n'arrive qu'en fin de PNMR2. De plus, leur dispositif de financement, tant dans les circuits que dans les moyens alloués, est très insuffisant.

Nous sommes dans la bonne direction, car des progrès ont été réalisés, mais trop lentement. Le déficit de réponses à apporter face aux besoins appelle à la poursuite d'une politique de santé volontariste pour les maladies rares.

\section{Quels enseignements pour améliorer l'avenir ?}

Alors que la première version du plan se composait de mesures précises et mesurables, le PNMR2 publié en février 2011 a été reformaté, en remplaçant un certain nombre de mesures opérationnelles par de simples déclarations d'intention. Pour y remédier, un réel pilotage adaptatif aurait dû être mis en place - cela n'a malheureusement pas été le cas. La mission de coordination et d'impulsion, qui devait être portée par le Secrétariat général du plan, n'a pas été remplie pendant une très longue période. Le PNMR mobilise de nombreux acteurs de natures très différentes. Le pilotage du plan ayant été essentiellement assuré par la DGOS, la coordination de ces acteurs n'a pas été facilitée. Plus globalement, la dimension interministérielle du plan (santé, recherche, secteur médico-social et industrie) a fait défaut. Pour un plan aussi transversal que celui des maladies rares, la mise en place d'une structure de gouvernance ad hoc, à la fois transversale et interministérielle aurait été nécessaire. En pratique, le Cospro n'a réalisé aucun travail prospectif. Comme il s'est limité à des réunions de présentation de l'état d'avancement des actions par les ministères, il n'a pas impulsé de logique transversale de travail en commun.

La mesure «Filières de santé » illustre parfaitement cette situation. Cette mesure aurait dû apparaître dans le plan comme une mesure chapeau des trois axes du plan puisque le but des filières est de mettre en interaction les différents acteurs. Comme les filières étaient pilotées par la seule DGOS et financées sur des budgets hospitaliers, l'association des acteurs a été rendue difficile, notamment ceux de la recherche et du médicament. La mobilisation des financements est un système kafkaïen avec, en sus, la question des frais de gestion prélevés par les hôpitaux, qui peuvent prélever jusqu'à $25 \%$ de frais de gestion.

\section{Points faibles et avancées positives}

Les centres de référence et de compétences ont permis d'améliorer la prise en charge des malades atteints de maladies rares. La lisibilité du système de santé a été améliorée. Les médecins de proximité connaissent mieux l'existence des centres de référence. La mise en interaction de l'ensemble des acteurs est un facteur d'amélioration globale. Les filières de santé, les centres de référence et le travail en commun des acteurs à travers les réseaux, les commissions des filières, les PNDS doivent être pérennisés. Les maladies rares nécessitent une politique nationale qui porte une vision stratégique et une réelle possibilité de pilotage assurant la transversalité entre des directions, des ministères et des acteurs publics et privés. Cela est rendu encore plus nécessaire à I'heure où la multiplication des pistes thérapeutiques et l'arrivée des premiers traitements dans le domaine des maladies rares deviennent réalité. C'est pourquoi nous appelons les pouvoirs publics - malheureusement peu représentés aujourd'hui - et l'ensemble des acteurs à se mobiliser dans un troisième PNMR ou a minima une véritable stratégie nationale sur les maladies rares pilotée par une structure de coordination réellement transversale et disposant de vrais moyens de fonctionnement.

\section{Didier Lacombe}

Le PNMR2 a souffert d'un manque de moyens financiers et de soutien politique. II faut savoir qu'aux États-Unis, le président Obama a annoncé que des fonds publics seraient alloués à la médecine personnalisée et au développement du séquençage haut débit de nouvelle génération. Toutefois, le PNMR2 offre un certain nombre d'éléments positifs, comme la mise en place des filières et la création de la Fondation maladies rares. $\diamond$

\section{ÉCHANGES AVEC LA SALLE}

\section{De la salle}

Quid du PNMR3?

\section{Didier Lacombe}

Nous n'avons aucune visibilité sur le futur plan.

\section{Hélène Dollfus}

Je partage totalement les propos de M. Cottet. L'avenir du PNMR doit dès à présent faire l'objet d'une réflexion. Les associations devraient se mobiliser pour pérenniser le plan sous une forme ou une autre. Plusieurs axes de réflexion ont 
été mentionnés, notamment en termes d'organisation, de moyens, etc. Pour l'avenir du plan, le modèle de l'Institut National du Cancer (INCa) est intéressant car il réunit autour d'une même table les soignants, les chercheurs et les tutelles. Ainsi, le modèle devrait être plus efficace et plus compact afin de prendre des décisions plus stratégiques et plus prospectives.

\section{Didier Lacombe}

Le rapprochement avec I'INCa est intéressant. L'évolution de la Fondation maladies rares vers une vraie structure d'État comme I'INCa - avec un financement pérenne - devrait être envisagée.

\section{Christian Cottet}

Au cours des deux PNMR, trois éléments structurants ont été mis en place : les centres de référence, la Fondation maladies rares et les filières de santé. Nous regrettons que les pouvoirs publics aient très peu financé la Fondation. Sans la générosité publique, cet organisme n'aurait pas fonctionné. La Fondation maladies rares a vocation à coordonner et impulser une recherche dans certaines thématiques. Une structure de type « institut des maladies rares » chargée de l'animation et de la gouvernance d'une politique nationale se situerait à un niveau supérieur.

\section{De la salle}

Quels sont les critères de sélection des centres de référence et de compétences? Comment sont-ils sélectionnés?

\section{Didier Lacombe}

Une autoévaluation est assurée par les directions d'hôpitaux, via un questionnaire en ligne. La visite sur site n'est plus obligatoire, sauf en cas de problème. Le label est accordé pour cinq ans. II appartient aux coordonnateurs des centres de référence de prouver la qualité de leur travail. Nous espérons que les bons centres de référence pourront continuer à travailler et obtenir des financements. L'ensemble des comités du PNMRI ayant été dissous, il est important via les comités de sélection de pouvoir créer de nouveaux centres de référence ou de faire évoluer certains centres de référence.

\section{Hélène Dollfus}

Remplir les rapports annuels d'activité en ligne est très chronophage. Cela donne toutefois l'occasion d'avoir une vraie discussion avec les directions des hôpitaux. Parmi les critères d'évaluation importants figurent le nombre de consultations, la formation, la communication en direction du grand public et l'articulation avec la recherche. La méthodologie PIRAMIG, utilisée par les MIG, a été adaptée aux maladies rares.

\section{Didier Lacombe}

Les crédits MIG et MERRI ont été réduits cette année de manière drastique dans tous les CHU de France.

\section{De la salle}

Pourquoi aucun représentant du ministère n'est-il présent aujourd'hui ?

\section{Didier Lacombe}

Nous avons lancé des invitations, mais elles ont été déclinées.

\section{De la salle}

Il faudrait davantage donner la parole aux acteurs de terrain.

\section{Didier Lacombe}

Les associations et les patients ont été les pièces mâ̂tresses des deux PNMR.

\section{Point de vue des industriels}

\section{Sylvain Forget}

Les maladies rares interrogent les industriels sur leur fonctionnement, leur positionnement et leur manière de conduire leur recherche. 15 ans après la mise en place des réglementations européennes, les malades sont mieux pris en charge, mais des progrès restent à réaliser.

Les industriels du médicament ont d'abord pour mission d'apporter des solutions thérapeutiques médicamenteuses. Près de 300 médicaments sont utilisés tous les jours en France pour le traitement de maladies rares. 85 d'entre eux ont le statut de médicament orphelin. Selon Orphanet, 115 médicaments avec AMM ont une indication pour une maladie rare sans avoir un statut d'orphelin. Depuis le règlement de 2000, plus de 1500 médicaments ont obtenu une désignation de médicament orphelin ; leur intérêt doit désormais être démontré pour obtenir une AMM.

Selon un récent rapport d'Evaluate pharma, $23 \%$ des investissements de phase III dans la recherche biomédicale par les industries du médicament sont portés sur les maladies rares. Ce pourcentage traduit un investissement très lourd en personnes et en recherche, en passant du modèle des blockbusters vers un modèle orienté vers la médecine à $4 \mathrm{P}$ (prédictive, préventive, personnalisée et en partenariat avec des acteurs allant au-delà des prescripteurs).

En France, les Autorisations Temporaires d'Utilisation (ATU) de cohorte et les ATU nominatives sont très importantes. Une bonne partie d'entre elles sont captées par les maladies rares. À ce jour, une centaine de médicaments seraient susceptibles de justifier une Recommandation Temporaire d'Utilisation (RTU). Après repositionnement et amélioration, ils pourraient obtenir une AMM. Avec près de 300 médicaments dédiés au traitement de maladies rares utilisés en France, nous pouvons considérer que les industriels du médicament font leur travail. L'ont-ils fait pour trop cher ? Le coût des médicaments dans notre système de santé est en train de diminuer. Les médicaments non orphelins relèvent du système classique ; leur coût est en train de baisser. Selon un récent rapport du Comité Économique des Produits de Santé (CEPS), les médicaments orphelins représentent un coût d'un peu moins d'un milliard d'euros, soit $4 \%$ du coût total des 
médicaments. Ces derniers représentant $15 \%$ des dépenses de santé, le coût des médicaments orphelins représente donc un peu moins de 0,6\% des dépenses de santé. Une hausse même de $10 \%$ du coût de ces médicaments ne risquerait donc pas de mettre en péril le système de la Sécurité sociale. En outre, l'exclusivité des médicaments orphelins s'arrêtant au bout de dix ans, les premiers génériques seront prochainement mis sur le marché. Ainsi, force est d'admettre que les industriels ont pratiqué une politique de coût compatible avec la continuité de notre système de prise en charge.

Jusqu'à présent, les industriels ont toujours essayé de développer des partenariats avec l'ensemble des acteurs du monde des maladies rares. Dans le cadre d'une plate-forme de communication qui sera ouverte en janvier 2016, ils formuleront une vingtaine de propositions réparties en quatre chapitres: la recherche, la simplification du labyrinthe administratif, le financement et les partenariats. S'agissant du dernier thème, nous considérons que le rôle des industriels ne se limite pas à fournir des boîtes de médicaments. En effet, les industriels sont prêts à participer encore plus activement aussi bien à l'évaluation du travail réalisé depuis une décennie qu'à la mise en place du PNMR3 qu'ils appellent de leurs vœux.

Le bilan que nous avons dressé des PNMR a mis en évidence un certain nombre d'éléments. La structuration apportée par les plans est très utile aux industriels. La mise en place de partenariats associatifspublic-privé doit être renforcée. La Fondation maladies rares, qui implique les industriels, doit être développée. La mise en place d'un modèle de prise en charge sociétale est à pérenniser et à amplifier. Les PNMR doivent jouer un rôle moteur pour l'Europe. Les industriels n'envisagent jamais de développer un médicament orphelin à l'échelle d'un seul pays. Le rôle des industriels dans les PNMR est à préciser et à renforcer. Enfin, le modèle économique doit être sécurisé.

\section{Didier Lacombe}

Le fait que les médicaments orphelins représentent $4 \%$ du coût des médicaments me convient bien puisque $4 \%$ de la population française serait concernée par les maladies rares. En tant que pédiatre, je suis de la génération des professionnels qui ont dépisté les enfants, à la naissance, de la mucoviscidose, qui les ont pris en charge et qui les ont vus mourir. Aujourd'hui, la synthèse de tous les médicaments développés dans la mucoviscidose ouvre une vraie porte d'espoir. La combinaison de ces médicaments devrait permettre aux médecins qui auront dépisté et suivi ces enfants de ne pas les voir décéder à un jeune âge adulte.

\section{Christian Cottet}

Depuis quelques années, l'industrie du médicament est en train d'expier le péché originel de ne pas s'être intéressée historiquement aux maladies rares. C'est très positif. Les chiffres cités par Sylvain Forget montrent la nécessité d'organiser cette politique de santé publique, dans la mesure où $98 \%$ des maladies rares n'ont pas de traitement aujourd'hui.

La France est pionnière en matière d'ATU et de RTU. Un vrai travail devra être mené, car les références ne sont pas forcément disponibles pour aller vers la mise en œuvre des RTU.

La question du modèle économique et du prix du médicament se pose. Les industriels doivent bien sûr être rentables. Le modèle économique classique n'est pas adapté aux maladies extrêmement rares. Nous devons faire preuve collectivement de créativité. Nous avons ainsi avancé le concept de « prix juste et maîtrisé ». Un travail abouti doit être mené dans ce domaine, car la question de la rentabilisation des efforts de recherche se pose.

\section{Marc Hanauer, Orphanet}

Fondé en 1997 par le Dr Ségolène Aymé, Orphanet a été l'un des acteurs fortement impactés par les PNMR qui ont permis de consolider ses activités principales, telles que la diffusion et la mise à disposition d'informations. S'il est peut-être passé inaperçu, l'axe du PNMR2 relatif à la nomenclature des maladies rares revêt néanmoins une grande importance. Celles-ci sont sous-représentées dans les systèmes d'information et les nomenclatures. Une note parue en 2012 a permis l'inclusion des maladies rares, notamment dans les systèmes d'information permettant de suivre les dossiers des patients dans les centres de référence. Ce sujet pose directement la question de l'interopérabilité entre les différents systèmes d'information et bases de données dans le cadre de la recherche.

Orphanet est aussi un consortium international. Toute stratégie discutée au niveau national doit tenir compte du contexte international. Le premier PNMR a joué un rôle moteur en Europe. Le futur PNMR3 devra s'inscrire dans une dynamique internationale.

\section{De la salle}

Quel est l'avenir du personnel embauché par les Filières Maladies Rares?

\section{Didier Lacombe}

Nous espérons que le financement des filières sera pérennisé, mais ce n'est pas acquis. En outre, des négociations avec les directions de CHU peuvent être engagées pour que ce personnel soit CDIsé.

\section{De la salle}

Comment pallier le manque d'information des médecins généralistes?

\section{Didier Lacombe}

La journée internationale des maladies rares qui se tiendra en février 2016 sera l'occasion d'organiser une information sur les centres de référence en tant que nouveaux moyens de diagnostic et d'étude du génome. Cette journée sera ouverte aux médecins et au grand public. $\diamond$

\section{LIENS D'INTÉRÊT}

D. Lacombe déclare participer à des interventions ponctuelles pour les entreprises Shire, Genzyme, Biomarin.

H. Dollfus, S. Forget, C. Cottet, M. Hanauer déclarent n'avoir aucun lien d'intérêt concernant les données publiées dans cet article. 